\title{
BMJ Open Chlorhexidine oral rinses for symptomatic COPD: a randomised, blind, placebo-controlled preliminary study
}

\author{
Alexa A Pragman (D) ,, Ann M Fieberg, ${ }^{3}$ Cavan S Reilly, ${ }^{4}$ Christine Wendt (D) ${ }^{1,2}$
}

To cite: Pragman AA, Fieberg AM, Reilly CS, et al. Chlorhexidine oral rinses for symptomatic COPD: a randomised, blind, placebo-controlled preliminary study. BMJ Open 2021;11:e050271. doi:10.1136/ bmjopen-2021-050271

- Prepublication history and additional supplemental material for this paper are available online. To view these files, please visit the journal online (http://dx.doi.org/10.1136/ bmjopen-2021-050271).

This work was previously presented virtually at IDWeek 2020 (21 October 2020).

Received 16 February 2021 Accepted 29 0ctober 2021

Check for updates

(C) Author(s) (or their employer(s)) 2021. Re-use permitted under CC BY-NC. No commercial re-use. See rights and permissions. Published by BMJ.

${ }^{1}$ Department of Medicine, Minneapolis VA Health Care System, Minneapolis, Minnesota, USA

${ }^{2}$ Department of Medicine, University of Minnesota Medical School, Minneapolis, Minnesota, USA

${ }^{3}$ Coordinating Center for Biometric Research, University of Minnesota, Minneapolis,

Minnesota, USA

${ }^{4}$ Division of Biostatistics, University of Minnesota, Minneapolis, Minnesota, USA

Correspondence to Dr Christine Wendt; wendt005@umn.edu

\section{ABSTRACT}

Objectives Determine the effect of twice-daily chlorhexidine oral rinses on oral and lung microbiota biomass and respiratory symptoms.

Setting Single centre.

Participants Participants were aged 40-85 with chronic obstructive pulmonary disease (COPD) and chronic productive cough or COPD exacerbation within the last year. Exclusions included antibiotics in the previous 2 months and/or those with less than four teeth. Forty-four participants were recruited and 40 completed the study.

Intervention Participants were randomised 1:1 to twicedaily $0.12 \%$ chlorhexidine oral rinses versus placebo for 2 months along with daily diaries. St. George's Respiratory Questionnaire (SGRQ), blood tests, oral rinse and induced sputum were collected at randomisation and the final visit. Primary and secondary outcomes Primary outcome was a change in oral and sputum microbiota biomass. Secondary outcomes included: sputum and oral microbiota Shannon and Simpson diversity and taxonomy; inflammatory markers; Breathlessness, Cough and Sputum Scale and SGRQ scores.

Results Neither the oral microbiota nor the sputum microbiota biomass decreased significantly in those using chlorhexidine compared with placebo (oral microbiota mean $\log _{10}$ difference $(\mathrm{SE})=-0.103(0.23)$, $95 \% \mathrm{Cl}-0.59$ to $0.38, \mathrm{p}=0.665$; sputum microbiota 0.80 (0.46), $95 \% \mathrm{Cl}-0.15$ to $1.75, \mathrm{p}=0.096)$. Chlorhexidine decreased both oral and sputum microbiota alpha (Shannon) diversity (linear regression estimate (SE) oral: -0.349 (0.091), $p=0.001$; sputum $-0.622(0.169)$, $\mathrm{p}=0.001)$. Chlorhexidine use did not decrease systemic inflammatory markers compared with placebo (C reactive protein (chlorhexidine $1.8 \pm 7.5$ vs placebo $0.4 \pm 6.8, p=0.467)$, fibrinogen $(22.5 \pm 77.8$ vs $10.0 \pm 77.0$, $p=0.406)$ or leucocytes $(0.2 \pm 1.8$ vs $0.5 \pm 1.8, p=0.560)$ ). Chlorhexidine use decreased SGRQ scores compared with placebo (chlorhexidine $-4.7 \pm 8.0$ vs placebo $1.7 \pm 8.9$, $\mathrm{p}=0.032$ ).

Conclusions We did not detect a significant difference in microbiota biomass due to chlorhexidine use.

Chlorhexidine decreased oral and sputum microbiota alpha diversity and improved respiratory health-related quality of life compared with placebo.

Trial registration NCT02252588.
Strengths and limitations of this study

- Using a randomised control design, this study will provide the first example of the effects of altering the oral microbiome in the setting of chronic obstructive pulmonary disease (COPD)

- A study intervention that is simple, inexpensive, and has few side effects.

- Our study was limited by its relatively small sample size and single-centre design.

- Other limitations include our inability to distinguish between live and dead bacteria in our samples.

\section{INTRODUCTION}

Chronic obstructive pulmonary disease (COPD) is the third-leading cause of death worldwide and a significant cause of morbidity and mortality. ${ }^{1}$ COPD symptoms such as chronic cough, sputum production, breathlessness and wheezing lead to decreased quality of life. COPD exacerbations are a major cause of this morbidity. Medications such as bronchodilators and anti-inflammatory medications modestly reduce COPD exacerbations but have not effectively improved symptoms as assessed by health status. Approximately $50 \%$ of COPD exacerbations are attributed to bacteria ${ }^{23}$ and patients with COPD often remain colonised with bacteria in their lower respiratory tracts even during periods of stable disease. ${ }^{3}$ These bacteria make up the lung microbiota. Recent evidence supports that the oral microbiota is the main source of the lung microbiota. ${ }^{45}$ The COPD lung microbiota also correlates with COPD exacerbation frequency. ${ }^{6}$ No studies have yet been conducted that seek to alter the COPD microbiota biomass using common and safe medications with only mild side effects.

Chlorhexidine is a topical antiseptic that is Food and Drug Administration-approved for use as an oral rinse. ${ }^{7}$ It binds to bacterial cell walls and exerts bacteriostatic and 
bacteriocidal effects; it is broadly active against Gram positive and Gram negative bacteria as well as yeasts. In oral rinses it reduces dental plaque, gingivitis, periodontitis and decreases oral bacteria after dental extractions or trauma. In meta-analysis, chlorhexidine oral rinses have been shown to reduce the risk of ventilator-associated pneumonia. ${ }^{8}$ It is well-tolerated, with known side effects consisting of mild oral discomfort, transient decrease in taste, and tooth discolouration (particularly with tea or coffee consumption).

Randomised controlled trials of chlorhexidine oral rinses for dental diseases have shown some possible decrease in oral bacterial biomass, ${ }^{910}$ decrease in specific oral pathogens ${ }^{10}$ and decreased alpha diversity of the oral microbiota. ${ }^{11}$ Oral chlorhexidine use results in an immediate and sustained decrease in oral bacteria viability. ${ }^{12}$

There is compelling evidence that chlorhexidine oral rinses improve oral health and are safe and well-tolerated. The oral microbiota is the source of the lung microbiota likely due to microaspiration. Among those with COPD, the oral and sputum microbiota correlate with COPD exacerbation frequency. ${ }^{6}$ Oral treatment with chlorhexidine alters the oral microbiota, which may subsequently alter the lung microbiota and COPD-related symptoms. Our primary aim was to determine the effect of twicedaily chlorhexidine oral rinses on oral and lung microbiota biomass in participants with COPD.

\section{METHODS}

The ChLorhexidine effect In the oral and lung MicroBiota study (CLIMB) is a randomised, blind, placebocontrolled, parallel-group preliminary study of the effects of chlorhexidine oral rinses on COPD. It was conducted at a single tertiary-care Veterans Affairs medical centre (USA). A data monitoring committee did not oversee the study. All data relevant to the study are included in the article. Protocol and additional methods are provided in an online data supplement, and the dataset is available in Dryad. ${ }^{13}$

\section{Patient and public involvement}

The design of this study was based on previous randomised clinical trials designed for COPD exacerbations. We further received input from expert clinicians and researchers within the COPD Clinical Research Network. Patients with COPD were not involved in the development of the protocol, but participant feedback was obtained during the study.

\section{Study protocol}

Eligible participants were invited to participate in the study and consisted of those aged 40-85 years with a diagnosis of COPD and the presence or high likelihood of a chronic cough and sputum production. Participants were excluded if they were not fully recovered for at least 30 days from a COPD exacerbation or were treated with antibiotics in the last 2 months.
Participants were assigned (1:1) via a random number generator to receive either $15 \mathrm{~mL}$ of twice-daily $0.12 \%$ oral chlorhexidine rinses (PerioGard) ${ }^{7}$ or matched placebo mouth rinses for 8 weeks. The pharmacist conducted the allocation and assignment and was the only staff member unblinded to study assignment. Study data were collected and managed using REDCap electronic data capture tools hosted at the University of Minnesota. ${ }^{1415}$

At visit 1, participants provided medical history, performed spirometry, completed the St. George's Respiratory Questionnaire (SGRQ) ${ }^{1617}$ were instructed on how to complete the Breathlessness, Cough and Sputum Scale $(\mathrm{BCSS})^{18}$ daily diaries, and provided blood, oral and induced sputum samples prior to randomisation. Oral and sputum sample volumes were recorded. Sputum production was heterogeneous across participants and samples, so sputum sample $16 \mathrm{~S}$ copy numbers were normalised to (ie, divided by) sputum sample mass. Oral sample size also varied due to variations in expectoration efficiency and were therefore also normalised to oral sample mass. Participants returned 8 weeks later to return BCSS diaries, complete the SGRQ assess outcomes, and provide blood, oral and sputum samples.

The clinical laboratories at the Minneapolis Veterans Affairs Medical Center determined white cell count (WBC) and differential, fibrinogen, $\mathrm{C}$ reactive protein (CRP) levels and sputum gram stain and culture results. All oral rinses, sputum samples, and unused sterile water (control samples) were frozen immediately and until DNA extraction. 16S rRNA quantification and 16S rRNA V4 MiSeq sequencing was performed at the University of Minnesota Genomics Center as previously described. ${ }^{19}$

\section{Outcomes and power analysis}

The primary outcome was change in oral and sputum microbiota biomass after 8 weeks of chlorhexidine versus placebo use, compared with baseline values as assessed by $16 \mathrm{~S}$ rRNA quantification. The primary outcome was chosen based on the mechanism of action of chlorhexidine, however, sample size calculations were based on a change in alpha diversity (a secondary outcome) due to data availability at study initiation. At a sample size of 20 per group and across a plausible range of effect sizes, our study had $67 \%-94 \%$ power to detect a change in alpha diversity associated with chlorhexidine use. Sample size calculations are available in online supplemental file 1 , and a rarefaction curve is provided in online supplemental figure S1. Secondary outcomes included: sputum and oral microbiota Shannon and Simpson diversity; sputum and oral microbiota taxonomy; inflammatory markers (WBC, fibrinogen and CRP); BCSS scores; SGRQ score and assessment of adverse events.

\section{Statistical analysis}

Baseline variables were compared using Fisher's exact test for categorical variables or the Wilcoxon two-sample test for continuous variables. Means are presented with SD; 
mean differences and parameter estimates are presented with their associated SE.

All analyses were performed using SAS V.9.4 (SAS Institute) and the modified-to-treat principle. A two-sided type I error of 0.05 was used. Correction of the type I error rate for multiple testing was performed using the Step-down Bonferroni method. ${ }^{20}$

For the primary analysis of both normalised oral wash and normalised sputum biomass count, values were transformed to the $\log _{10}$ scale and the mean difference between treatment groups was compared using the twosample t-test. A multiple imputation procedure was used to impute each unavailable sputum weight.

The primary data analysis included all those who completed the study, with baseline and mid-study phone call data included for non-completers when available. A subanalysis of the microbiota data was conducted after excluding samples obtained from participants who used antibiotics during the study period.

Linear regression was used to examine the effect of treatment group on the 8-week change in the Shannon and Simpson biodiversity indices, BCSS, SGRQ and inflammatory markers separately, with each model adjusted for the baseline value of the measure.

Subgroup analyses of participants who did not receive antibiotics during the study were also performed for the outcomes of biomass and biodiversity.

For taxa abundance analyses, treatment effects on abundance were examined by modelling the 8-week change using linear regression, adjusted for baseline count. Analysis was restricted to genera with $<20 \%$ of values equal to zero. Fisher's exact test was used to determine the proportion with a genus detected at week 8 versus baseline compared between treatment groups. Results were corrected for multiple comparisons.

\section{RESULTS}

CLIMB assessed 511 participants for eligibility, excluded 215 because they did not meet criteria, 252 declined to participate and 44 were randomised to study medication. Participants were recruited between 8 September 2014 and 30 May 2019 and the study ended when 40 participants completed the 8-week study. Four participants (all randomised to chlorhexidine) discontinued the study, leaving 20 participants in each group who completed the study. One participant withdrew without using any study medication, while the other three were lost to follow-up (figure 1).

Of the 44 CLIMB participants, $41(93 \%)$ were male and $42(95 \%)$ were Caucasian. The mean age was 67.9 years and mean tobacco exposure was 58.2 pack-years. Most were former tobacco users $(31,70 \%)$ and the remainder were current smokers. High blood pressure $(31,70 \%)$ and coronary artery disease $(27,61 \%)$ were reported by most participants. Mean forced expiratory volume in $1 \mathrm{~s}$ $\left(\mathrm{FEV}_{1}\right) \%$ predicted $\left(\mathrm{FEV}_{1} \mathrm{pp}\right)$ was $41.7 \%$ and the mean number of COPD exacerbations reported in the prior 12

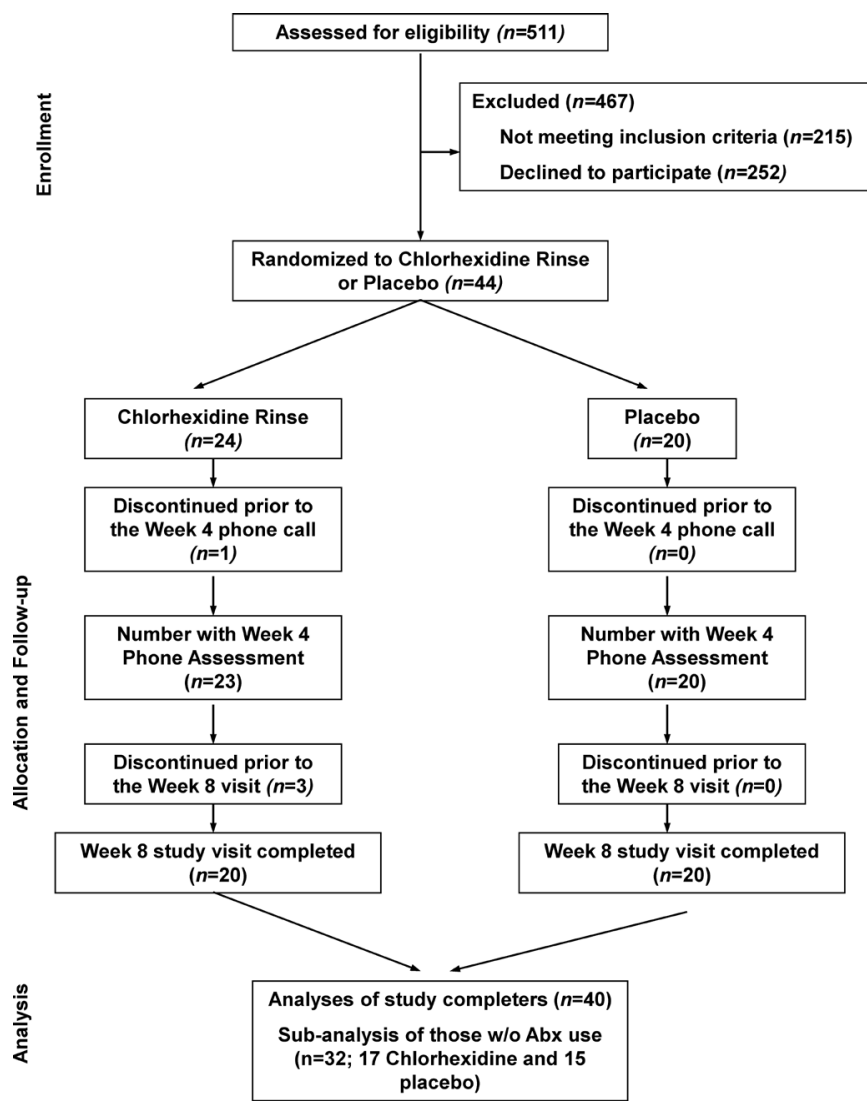

Figure 1 ChLorhexidine effect In the oral and lung MicroBiota (CLIMB) study consort diagram. CLIMB assessed 511 individuals for eligibility. Of these, 467 were excluded and 44 were randomised. Four participants (all assigned to the chlorhexidine group) discontinued the study. Forty participants completed the study.

months was 2.1. Baseline mean SGRQ score was 45.8. No baseline characteristics differed significantly by treatment group (table 1).

The number of participants experiencing a COPD exacerbation or using an antibiotic or oral corticosteroid during the study period are presented in table 2 . Eight participants (3 in the chlorhexidine group, 5 in the placebo group) received antibiotics during the study; most but not all antibiotic use was for a respiratory indication. No participants experienced more than one exacerbation, more than one course of antibiotics or more than one course of oral corticosteroids during the study.

Our primary outcome was a change in oral and sputum microbiota biomass during the study period as assessed by $16 \mathrm{~S}$ rRNA copy numbers. Oral rinse samples were available for 40 participants (20 per group). There was a decrease in biomass in both groups; the mean \pm SD changes were $-0.24 \pm 1.0$ and $-0.14 \pm 0.32$ in the chlorhexidine and placebo groups, respectively (online supplemental table S1). The mean difference between treatment groups (active-placebo) was not significant (mean diff $(\mathrm{SE})=-0.103$ (0.23), 95\% CI -0.59 to 0.38 , $\mathrm{p}=0.665)$. Very similar results were seen in the subgroup that did not use antibiotics during the study $(\mathrm{N}=32$, 
Table 1 Baseline characteristics by treatment group

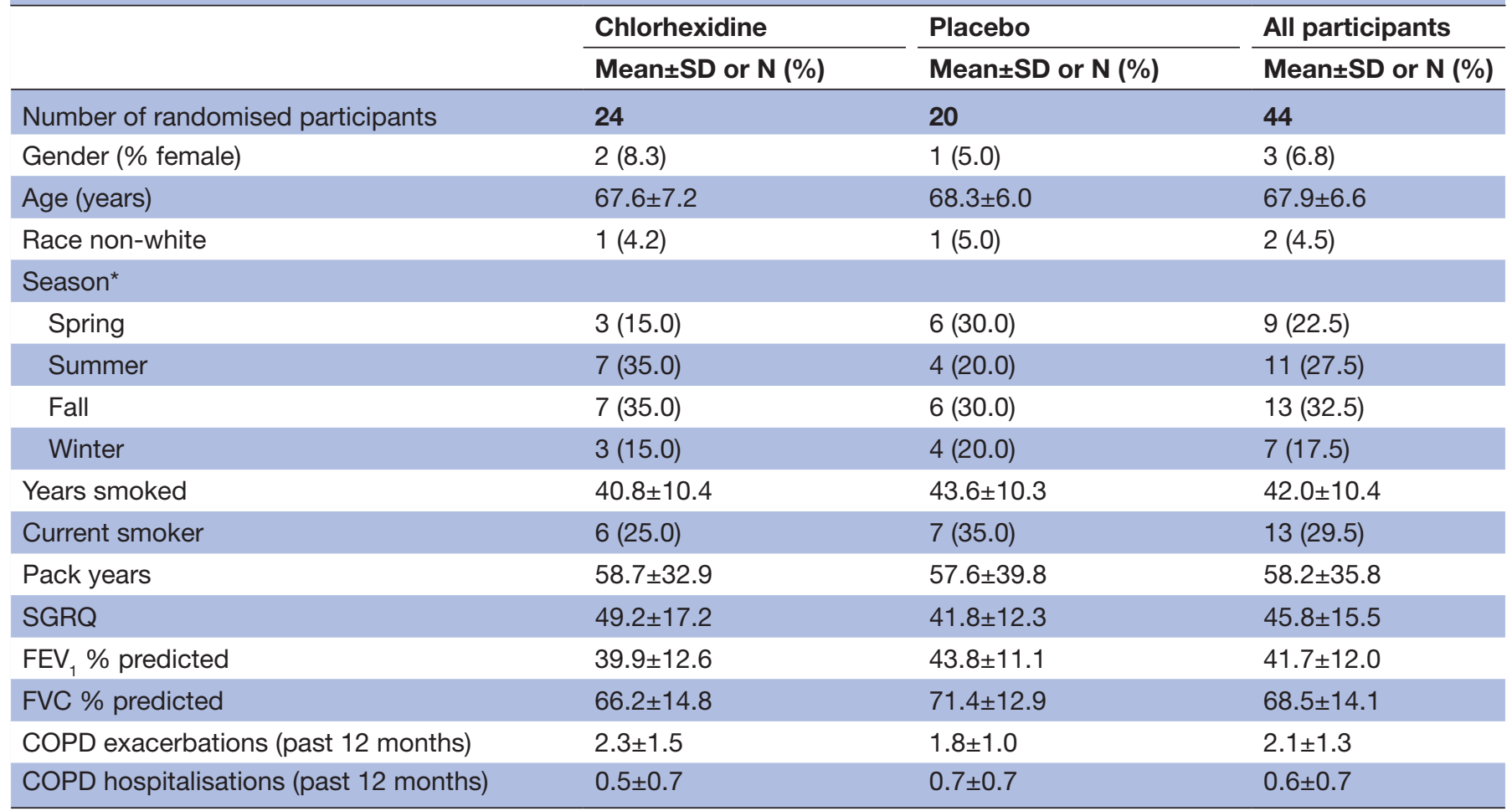

${ }^{*}$ Assigned to the season that covered $>50 \%$ of the study period for a given participant.

COPD, chronic obstructive pulmonary disease; $F_{1}$, forced expiratory volume in 1 s; FVC, forced vital capacity; SGRQ, St. George's

Respiratory Questionnaire.

mean diff $(\mathrm{SE})=-0.07(0.29), 95 \%$ CI -0.65 to 0.51 , $\mathrm{p}=0.808$ ) (table 3).

For the analysis of biomass in sputum samples, five chlorhexidine and four placebo participants were unable to provide sputum samples; two were unable at baseline, six were unable at week 8 (including the four withdrawals) and one was unable at both baseline and week

Table 2 Exacerbations, antibiotic use or systemic steroid use during the study, excluding those withdrawn prior to study completion

\begin{tabular}{|c|c|c|}
\hline & Chlorhexidine & Placebo \\
\hline & $\mathbf{N}(\%)$ & N (\%) \\
\hline $\begin{array}{l}\text { Randomised participants } \\
\text { assessed ( } n)\end{array}$ & 20 & 20 \\
\hline COPD exacerbation* & $3(15.0)$ & $5(25.0)$ \\
\hline Systemic steroid use $†$ & $1(5.0)$ & $5(25.0)$ \\
\hline Antibiotic use $\ddagger$ & $3(15.0)$ & $5(25.0)$ \\
\hline
\end{tabular}

*Self-reported COPD exacerbation (worsening of chronic respiratory symptoms) during the study. One placebo subject reported an exacerbation but deferred any therapy until after study completion.

†Self-reported use of systemic corticosteroids during the study for any indication.

$\ddagger$ Self-reported use of systemic antibiotics during the study for any indication. One placebo subject took antibiotics for a nonrespiratory reason.

COPD, chronic obstructive pulmonary disease.
8. Among the 35 sputum samples, there were 11 missing sputum weight values ( 6 at baseline, 5 at week 8 ) among 4 placebo and 4 chlorhexidine participants. Table 3 shows the primary analysis results using a two-sample t-test with the normalised data available $(\mathrm{N}=27)$ and using a multiple imputation procedure to estimate the missing sputum weights $(\mathrm{N}=35)$. The two analysis methods provide similar results. Although we hypothesised that the estimate would be negative, indicating that the active group saw a larger decrease in biomass from baseline to week 8 than the placebo group, without imputation we see a non-significant effect in the opposite direction (mean $\log _{10}$ difference $(\mathrm{SE})=0.80 \quad(0.46), 95 \%$ CI -0.15 to $1.75, \mathrm{p}=0.096$ ) and similarly with imputation (mean $\log _{10}$ difference (SE) $=0.70$ (0.39), $95 \%$ CI -0.08 to 1.47 , $\mathrm{p}=0.078$ ). These results were supported by the subgroup analyses of those without antibiotic use during the study. Although the $p$ value for the imputation analysis is significant $(p=0.036)$ and the effect is not in the hypothesised direction, this result should be interpreted with caution due to the large number of tests reviewed here.

Linear regression was used to examine the 8-week change in each biodiversity index (Shannon and Simpson Indices) as a function of treatment group and adjusted for the value of the index at baseline (table 4). As hypothesised, those in the chlorhexidine group saw, on average, a significant decrease in the diversity indices in comparison to those in the placebo group. For the oral wash samples, those in the treatment group had a coefficient 
Table 3 Biomass analysis results - two-sample t-test on the $\log _{10}$ change

\begin{tabular}{|c|c|c|c|c|c|c|c|}
\hline $\begin{array}{l}\text { Two sample t-test on } \log _{10} \text { change } \\
\text { (CHL-PLA) }\end{array}$ & $\begin{array}{l}\text { N } \\
\text { TOT }\end{array}$ & $\begin{array}{l}\mathbf{N} \\
\mathrm{CHL}\end{array}$ & $\begin{array}{l}\mathbf{N} \\
\text { PLA }\end{array}$ & $\begin{array}{l}\text { Mean difference } \\
(\mathrm{SE})^{*}\end{array}$ & $95 \% \mathbf{C I}$ & $\mathbf{T}$ value & $P$ value \\
\hline \multicolumn{8}{|l|}{ All participants with available data } \\
\hline Oral wash samples & 40 & 20 & 20 & $-0.103(0.23)$ & -0.59 to 0.38 & -0.44 & 0.665 \\
\hline \multicolumn{8}{|l|}{ Sputum samples } \\
\hline No imputation & 27 & 15 & 12 & $0.80(0.46)$ & -0.15 to 1.75 & 1.73 & 0.096 \\
\hline Imputation† & 35 & 19 & 16 & $0.70(0.39)$ & -0.08 to 1.47 & 1.76 & 0.078 \\
\hline \multicolumn{8}{|l|}{ Excluding antibiotic use during study } \\
\hline Oral wash samples & 32 & 17 & 15 & $-0.07(0.29)$ & -0.65 to 0.51 & -0.25 & 0.808 \\
\hline \multicolumn{8}{|l|}{ Sputum samples } \\
\hline No imputation & 23 & 14 & 9 & $1.06(0.53)$ & -0.05 to 2.17 & 1.99 & 0.060 \\
\hline Imputation† & 28 & 16 & 12 & $0.98(0.47)$ & 0.06 to 1.89 & 2.09 & 0.036 \\
\hline
\end{tabular}

${ }^{*}$ Chlorhexidine group change in biomass minus placebo group.

†Imputation refers to the use of multiple imputation techniques to impute the 11 missing sputum weights.

CHL, chlorhexidine; PLA, placebo; TOT, total.

of -0.349 ( $\mathrm{SE}=0.091$, adj. $\mathrm{p}=0.001)$ for the Shannon diversity index and -0.030 ( $\mathrm{SE}=0.008$, adj. $\mathrm{p}=0.001$ ) for the Simpson diversity index. The results were similar for sputum samples: -0.622 ( $\mathrm{SE}=0.169$, adj. $\mathrm{p}=0.001)$ for the Shannon diversity index and $-0.091 \quad(\mathrm{SE}=0.034$, adj. $\mathrm{p}=0.0123$ ) for the Simpson diversity index. Very similar results for both oral wash and sputum alpha diversity were seen in the subgroup that did not use antibiotics during the study, indicating that the decrease in diversity with chlorhexidine use was not related to antibiotic use (online supplemental table S2).

For the additional secondary outcomes, the effect of treatment group on the 8-week change was examined using linear regression, adjusted for the measure at baseline (table 5 and online supplemental table S3). There was no significant difference between treatment groups over the 8-week study period in BCSS score (mean change in the chlorhexidine and placebo groups, respectively \pm SD: $-0.3 \pm 1.9$ vs $-0.1 \pm 1.5$, estimate $(95 \% \mathrm{CI})-0.28$ ( -1.45 to $0.89), \mathrm{p}=0.630)$, CRP $(1.8 \pm 7.5$ vs $0.4 \pm 6.8,1.54 \quad(-2.72$ to 5.80$), \mathrm{p}=0.467)$, fibrinogen $(22.5 \pm 77.8$ vs $10.0 \pm 77.0$, $20.19(-28.52$ to 68.91$), \mathrm{p}=0.406)$ or leucocytes $(0.2 \pm 1.8$ vs $0.5 \pm 1.8,-0.32(-1.42$ to 0.78$), \mathrm{p}=0.560)$. Participants in the chlorhexidine group showed a significantly larger decrease in SGRQ total score when compared with the placebo group (mean change $\pm \mathrm{SD}$ : $-4.7 \pm 8.0$ vs $1.7 \pm 8.9$, -6.22 ( -11.87 to -0.57$), \mathrm{p}=0.032)$. This difference was not evidenced in any one SGRQ domain.

In exploratory analyses, we evaluated the taxonomic composition of samples to assess for chlorhexidineassociated changes in the microbiota. Among sputum samples there were 42 genera. The results of the linear regression analyses showed that only Corynebacterium sequences were less abundant after chlorhexidine use

Table 4 Linear regression results of the effect of treatment group on the change in biodiversity

\begin{tabular}{|c|c|c|c|c|}
\hline \multirow[b]{2}{*}{ Outcome } & \multicolumn{3}{|l|}{ Linear regression } & \multirow{2}{*}{$\begin{array}{l}\text { Adjusted P } \\
\text { value }^{*}\end{array}$} \\
\hline & Predictor & Estimate (SE) & Unadjusted $\mathbf{P}$ value & \\
\hline \multicolumn{5}{|c|}{ Shannon diversity index change (week 8 -baseline) } \\
\hline \multirow[t]{2}{*}{ Oral wash $(\mathrm{N}=40)$} & Treatment group $\dagger$ & $-0.349(0.091)$ & 0.0005 & 0.0010 \\
\hline & Baseline index & $-0.197(0.073)$ & 0.0100 & \\
\hline \multirow[t]{2}{*}{ Sputum $(\mathrm{N}=35)$} & Treatment group & $-0.622(0.169)$ & 0.0008 & 0.0010 \\
\hline & Baseline index & $-0.312(0.111)$ & 0.0083 & \\
\hline \multicolumn{5}{|c|}{ Simpson diversity index change (week 8 -baseline) } \\
\hline \multirow[t]{2}{*}{ Oral wash $(\mathrm{N}=40)$} & Treatment group & $-0.030(0.008)$ & 0.0005 & 0.0010 \\
\hline & Baseline index & $-0.196(0.114)$ & 0.0938 & \\
\hline \multirow[t]{2}{*}{ Sputum $(\mathrm{N}=35)$} & Treatment group & $-0.091(0.034)$ & 0.0123 & 0.0123 \\
\hline & Baseline index & $-0.109(0.179)$ & 0.5472 & \\
\hline
\end{tabular}

*A step-down Bonferroni $p$ value adjustment is made for the two comparisons (oral wash and sputum) within each diversity index. $\dagger$ Treatment group is coded as chlorhexidine $=1$, placebo $=0$. 
Table 5 Linear regression analysis of the effect of treatment group on secondary outcomes

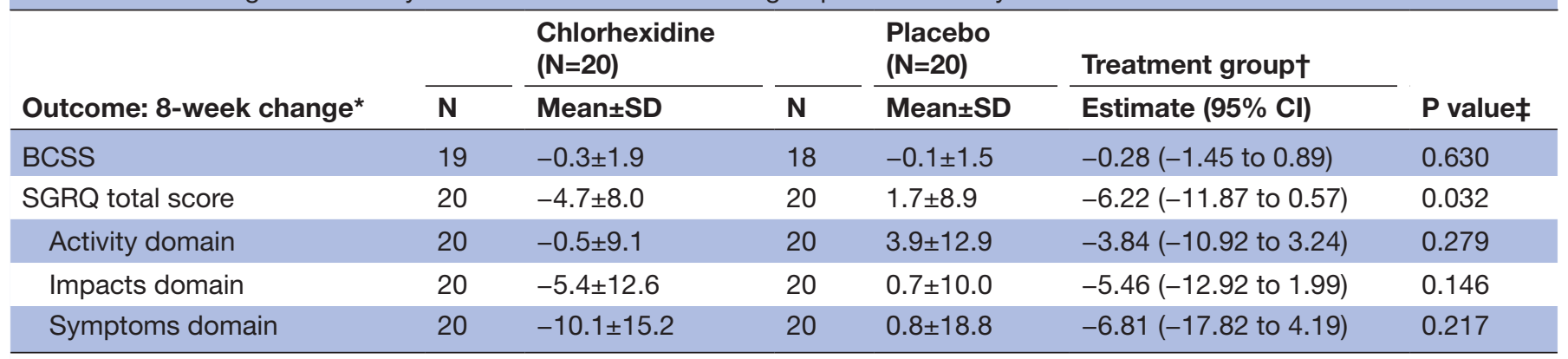

${ }^{*}$ Each model is adjusted for the baseline value of each outcome.

$\dagger$ Treatment group is coded as chlorhexidine $=1$, placebo $=0$.

$\ddagger$ The $p$-value is for the comparison of chlorhexidine versus placebo.

BCSS, Breathlessness, Cough and Sputum Scale; SGRQ, St. George's Respiratory Questionnaire.

compared with placebo (mean change in the chlorhexidine and placebo groups, respectively \pm SD: $-197 \pm 342$ vs $12 \pm 337$, estimate $(95 \%$ CI $)-282$ ( -438 to -126$)$, adjusted $\mathrm{p}=0.0378$ ). Among oral wash samples there were 43 genera. Only Lachnoanaerobaculum sequences were less abundant after chlorhexidine use compared with placebo (mean change in the chlorhexidine and placebo groups, respectively \pm SD: $-313 \pm 483$ vs $216 \pm 509$, estimate $(95 \%$ CI) -521 ( -815 to -226$)$, adjusted $\mathrm{p}=0.043)$. Follow-up analyses relying on the presence or absence of sequences (rather than relative abundance) produced similar results.

Very few adverse events were experienced over the course of the study (online supplemental table S4).

\section{DISCUSSION}

In this preliminary study, twice-daily chlorhexidine oral rinses decreased oral and sputum microbiota alpha diversity and improved pulmonary disease-related quality of life compared with placebo among those with symptomatic COPD. Chlorhexidine oral rinses did not appear to decrease the oral or sputum microbiota biomass, our primary outcome, compared with placebo as assessed by normalised $16 \mathrm{~S}$ rRNA quantitative PCR. Furthermore, during the 8-week treatment period chlorhexidine did not appear to decrease systemic inflammation or COPD symptoms, as assessed by the BCSS, compared with placebo. Our preliminary study had limited statistical power to detect several of our secondary endpoints; therefore, our results cannot definitively exclude a relationship between chlorhexidine use and systemic inflammation or symptoms.

We chose a change in biomass as our primary endpoint as we hypothesised that twice daily chlorhexidine would have its largest effect on microbiota biomass. However, we did not detect a significant decrease in biomass as a result of chlorhexidine use using quantitative PCR. Chlorhexidine is known to be bactericidal and previous work has identified a decrease in viable bacteria following chlorhexidine oral use compared with water. ${ }^{12}$
Our total DNA extraction technique coupled with PCRbased biomass determination is unable to distinguish between live and dead bacteria. It is therefore possible that chlorhexidine decreased the number of live bacteria in the oral and sputum microbiota, and that our PCRbased biomass determination technique was unable to distinguish between live bacterial biomass and dead bacteria. Furthermore, both groups experienced some decrease in biomass during the study period. Changes in dental care habits, including twice-daily oral rinsing with either study drug or placebo, may be responsible for this decrease.

Although total microbiota biomass did not appear to change, oral and sputum microbiota alpha diversity decreased as a result of chlorhexidine use. The healthy lung and oral microbiota generally demonstrate greater alpha diversity than the microbiota found in disease states such as COPD or cystic fibrosis. ${ }^{21}$ Whether this association is due to frequent use of antibiotics among those with chronic lung disease or due to the chronic lung disease itself remains unknown. Loss of alpha diversity due to chlorhexidine use may seem paradoxical given our current understanding of the relationship between low alpha diversity and worsening lung symptoms, however, the current disease model does not differentiate between alpha diversity per se and the mechanisms by which it may be manipulated. Loss of alpha diversity due to chlorhexidine use, antibiotic use or chronic lung inflammation likely represent clinically distinct entities.

Use of chlorhexidine oral rinses versus placebo did not result in decreased systemic inflammation as evidenced by CRP, fibrinogen and WBC values. These three systemic markers of inflammation are often elevated among those with symptomatic COPD. ${ }^{22}$ In light of our other findings linking chlorhexidine use to microbiota alterations and improved respiratory-related quality of life, we had expected that chlorhexidine use would lead to decreased systemic inflammation. It is possible that chlorhexidine use improved local inflammation (in the lungs or mouth) without resulting in systemic inflammatory changes. 
Sustained use over a longer time period may be needed in order to observe systemic anti-inflammatory effects.

Although chlorhexidine use did not result in significant changes to BCSS scores, respiratory health-related quality of life did improve with use of chlorhexidine oral rinses versus placebo during the 8-week intervention. SGRQ scores improved significantly among the chlorhexidine group relative to the placebo group, with a mean improvement (4.7 points) that is clinically meaningful (minimum clinically important difference of 4 points). The SGRQ encompasses three subscores for activity, impacts and symptoms. ${ }^{16}$ No subscore reached statistical significance, indicating that chlorhexidine use improved quality of life broadly, and was not due to isolated improvements in one or two SGRQ subdomains. Our data support the further study of chlorhexidine oral rinses among symptomatic patients with COPD to improve respiratory health-related quality of life.

In an exploratory analysis of the effects of chlorhexidine use on the sputum and oral microbiota, the only genus-level changes in DNA abundance were a decrease in Corynebacterium in sputum and a decrease in Lachnoanaerobaculum in oral rinses. Chlorhexidine is known to broadly decrease the viability of bacteria and yeast. ${ }^{12}$ Our microbiota analysis techniques, which cannot differentiate between DNA from 'live' or 'dead' organisms, therefore may be relatively insensitive to the effects of chlorhexidine. We were unable to detect overall changes in bacterial biomass or broad changes to individual genera among those using chlorhexidine compared with placebo. It is possible that broader assessments of the community composition, such as alpha diversity, are better able to detect chlorhexidine-related changes.

Our preliminary study had several strengths and limitations. Its strengths include a study intervention that is simple, inexpensive, and has few side effects; the randomised and blinded nature of the study; and objective assessment of outcomes. Our study was limited by its relatively small sample size and use of a secondary endpoint to determine statistical power, our homogeneous patient population and our single-centre design. In addition, other limitations include our inability to distinguish between live and dead bacteria in our samples, incomplete sample weights, lack of assessment of local inflammation, and limited in-person follow-up while on study drug. Future larger clinical trials will determine if the beneficial effects of chlorhexidine oral rinses can be sustained among COPD subjects, and the biological mechanisms for these improvements in quality of life.

Although we did not find a difference in daily respiratory symptoms as measured with the BCSS, we found a significant improvement in quality of life as measured by the SGRQ. This potential discrepancy likely arose because BCSS focuses solely on respiratory symptoms, ${ }^{18}$ while the SGRQ also assesses the broader impacts of COPD symptoms on quality of life. ${ }^{16}$ There was no single domain within the SGRQ that drove this result, but there was improvement in both the impacts and symptoms domains. We propose that oral chlorhexidine rinses improve respiratory health-related quality of life by decreasing the number of live oral bacteria, altering the content of the live oral microbiota, or both. Changes to the oral microbiota may decrease the lung inflammation that occurs following aspiration or change the composition of the lung microbiota itself and lead to an improved sense of wellness.

An additional clinical trial is needed to confirm our clinical endpoint findings with a larger group of participants and evaluate the mechanistic links between chlorhexidine, viable bacterial biomass, the microbiota and respiratory health-related qualify of life in symptomatic patients with COPD.

Our data indicate that the use of twice-daily chlorhexidine oral rinses among symptomatic patients with COPD improves quality of life. This was a secondary outcome in our study and warrants validation in a larger clinical trial. Our intervention is relatively easy to implement, inexpensive and well-tolerated.

Acknowledgements We gratefully acknowledge Ms Susan Johnson (study coordinator), Mr Shane Hodgson and Ms Allison Zank (laboratory technicians), Dr Douglas DeCarolis (research pharmacist) and Mr Trevor J Gould (bioinformatician). The views expressed in this article are those of the authors and do not reflect the views of the US Government, the Department of Veterans Affairs, the funders, the sponsors or any of the authors' affiliated academic institutions. Study data were collected and managed using REDCap electronic data capture tools hosted at the University of Minnesota. REDCap (Research Electronic Data Capture) is a secure, web-based software platform designed to support data capture for research studies, providing (1) an intuitive interface for validated data capture; (2) audit trails for tracking data manipulation and export procedures; (3) automated export procedures for seamless data downloads to common statistical packages and (4) procedures for data integration and interoperability with external sources.

Contributors AAP supervised research laboratory work, critically reviewed the data analyses and wrote the first draft with input from AMF. AMF performed the statistical analyses with supervision from CSR, contributed to the first draft and created the figure and tables. CSR supervised the statistical analyses and critically reviewed the manuscript. CW obtained funding, supervised subject recruitment, critically reviewed the data analysis and critically reviewed the manuscript. CW is the guarantor of the content of the manuscript, including the data and analysis.

Funding Flight Attendant Medical Research Institute (FAMRI) Clinical Innovator Award (\#150014 to CHW); Department of Veterans Affairs Office of Research and Development (1IK2CX001095 to AAP).

Competing interests None declared.

Patient consent for publication Not applicable.

Ethics approval Ethics approval was granted by the Minneapolis Veterans Affairs Medical Centre (MVAMC) Institutional Review Board (\#4526-A), all participants provided written consent, and all procedures adhered to the study protocol.

Provenance and peer review Not commissioned; externally peer reviewed.

Data availability statement Data are available in a public, open access repository. Extra data can be accessed via the Dryad data repository at http://datadryad.org/ with the doi:10.5061/dryad.m0cfxpp37.

Supplemental material This content has been supplied by the author(s). It has not been vetted by BMJ Publishing Group Limited (BMJ) and may not have been peer-reviewed. Any opinions or recommendations discussed are solely those of the author(s) and are not endorsed by BMJ. BMJ disclaims all liability and responsibility arising from any reliance placed on the content. Where the content includes any translated material, BMJ does not warrant the accuracy and reliability of the translations (including but not limited to local regulations, clinical guidelines, terminology, drug names and drug dosages), and is not responsible for any error and/or omissions arising from translation and adaptation or otherwise. 
Open access This is an open access article distributed in accordance with the Creative Commons Attribution Non Commercial (CC BY-NC 4.0) license, which permits others to distribute, remix, adapt, build upon this work non-commercially, and license their derivative works on different terms, provided the original work is properly cited, appropriate credit is given, any changes made indicated, and the use is non-commercial. See: http://creativecommons.org/licenses/by-nc/4.0/.

\section{ORCID iDs}

Alexa A Pragman http://orcid.org/0000-0001-8152-3951

Christine Wendt http://orcid.org/0000-0002-0924-8745

\section{REFERENCES}

1 WHO. The top 10 causes of death. Available: https://www.who.int/ news-room/fact-sheets/detail/the-top-10-causes-of-death [Accessed 12 Jul 2020].

2 Sethi S, Maloney J, Grove L, et al. Airway inflammation and bronchial bacterial colonization in chronic obstructive pulmonary disease. $\mathrm{Am}$ J Respir Crit Care Med 2006;173:991-8.

3 Sethi S, Murphy TF. Bacterial infection in chronic obstructive pulmonary disease in 2000: a state-of-the-art review. Clin Microbiol Rev 2001;14:336-63.

4 Pragman AA, Lyu T, Baller JA, et al. The lung tissue microbiota of mild and moderate chronic obstructive pulmonary disease. Microbiome 2018;6:e47305.

5 Bassis CM, Erb-Downward JR, Dickson RP, et al. Analysis of the upper respiratory tract microbiotas as the source of the lung and gastric microbiotas in healthy individuals. mBio 2015;6:e00037.

6 Pragman AA, Knutson KA, Gould TJ, et al. Chronic obstructive pulmonary disease upper airway microbiota alpha diversity is associated with exacerbation phenotype: a case-control observational study. Respir Res 2019;20:114.

773695 PerioGard Prntlbl. Available: https://www.google.com/url? sa $=t \& r c t=j \& q=\& e s r c=s \&$ source=web\&cd=\&ved=2ahUKEwj5uKe M3IzsAhXYWcOKHS4nCHYQFjAAegQIBBAB\&url= [Accessed $12 \mathrm{Jul}$ 2020].

8 Hua F, Xie H, Worthington HV, et al. Oral hygiene care for critically ill patients to prevent ventilator-associated pneumonia. Cochrane Database Syst Rev 2016;10:CD008367.
9 Becerik S, Türkoğlu O, Emingil G, et al. Antimicrobial effect of adjunctive use of chlorhexidine mouthrinse in untreated gingivitis: a randomized, placebo-controlled study. APMIS 2011;119:364-72.

10 Persson GR, Yeates J, Persson RE, et al. The impact of a lowfrequency chlorhexidine rinsing schedule on the subgingival microbiota (the teeth clinical trial). J Periodontol 2007;78:1751-8.

11 Al-Kamel A, Baraniya D, Al-Hajj WA, et al. Subgingival microbiome of experimental gingivitis: shifts associated with the use of chlorhexidine and $\mathrm{N}$-acetyl cysteine mouthwashes. J Oral Microbiol 2019;11:1608141.

12 Quintas V, Prada-López I, Donos N, et al. In situ neutralisation of the antibacterial effect of $0.2 \%$ Chlorhexidine on salivary microbiota: Quantification of substantivity. Arch Oral Biol 2015;60:1109-16.

13 Pragman AA, Fieberg A, Reilly CS. Data from: chlorhexidine oral rinses for symptomatic COPD: a randomized, blind, placebocontrolled preliminary study. Dryad Digital Repository 2021.

14 Harris PA, Taylor R, Thielke R, et al. Research electronic data capture (REDCap)--a metadata-driven methodology and workflow process for providing translational research informatics support. J Biomed Inform 2009;42:377-81.

15 Harris PA, Taylor R, Minor BL, et al. The REDCap Consortium: building an international community of software platform partners. $J$ Biomed Inform 2019;95:103208.

16 Jones PW, Quirk FH, Baveystock CM. The St George's respiratory questionnaire. Respir Med 1991;85 Suppl B:25-31. discussion 33.

17 Jones PW. St. George's respiratory questionnaire: MCID. COPD 2005;2:75-9.

18 Leidy NK, Rennard SI, Schmier J, et al. The breathlessness, cough, and sputum scale: the development of empirically based guidelines for interpretation. Chest 2003;124:2182-91.

19 Pragman AA, Knutson KA, Gould TJ, et al. Chronic obstructive pulmonary disease upper airway microbiome is associated with select clinical characteristics. PLoS One 2019;14:e0219962.

20 Holm S. A simple sequentially rejective multiple test procedure. Scand J Stat 1979;6:65-70.

21 Segal LN, Rom WN, Weiden MD. Lung microbiome for clinicians. New discoveries about bugs in healthy and diseased lungs. Ann Am Thorac Soc 2014;11:108-16.

22 Celli BR, Locantore N, Yates J, et al. Inflammatory biomarkers improve clinical prediction of mortality in chronic obstructive pulmonary disease. Am J Respir Crit Care Med 2012;185:1065-72. 